\title{
Comparison of Indirect Calorimetry and Common Prediction Equations for Evaluating Changes in Resting Metabolic Rate Induced by Resistance Training and a Hypercaloric Diet
}

CHRISTIAN RODRIGUEZ, PATRICK S. HARTY, MATTHEW T. STRATTON, MADELIN R. SIEDLER, ROBERT W. SMITH, BAYLOR A. JOHNSON, JACOB R. DELLINGER, ABEGALE D. WILLIAMS, SARAH J. WHITE, MARQUI L. BENAVIDES, GRANT M. TINSLEY

Energy Balance \& Body Composition Laboratory; Department of Kinesiology \& Sport Management; Texas Tech University; Lubbock, TX

Category: Masters

Advisor / Mentor: Tinsley, Grant (grant.tinsley@ttu.edu)

\section{ABSTRACT}

An individual's resting metabolic rate (RMR) is commonly the largest contributor to total daily energy expenditure. Prediction equations are most often employed by practitioners to estimate RMR, due to their superior practicality in many settings relative to laboratory methods like indirect calorimetry (IC). The ability to quantify RMR change over time may be more valuable than cross-sectional estimates as practitioners can then utilize these changes to prescribe adjustments to one's nutritional intake. PURPOSE: The purpose of this study was to assess the validity of several commonly used prediction equations to track RMR changes during a hypercaloric nutrition intervention and supervised exercise training program. METHODS: Twenty generally healthy males (mean \pm standard deviation; age: $21.9 \pm 2.6$ years; height: $178.1 \pm 6.9 \mathrm{~cm}$; body mass: $72.2 \pm 7.3 \mathrm{~kg}$; fat-free mass index: $18.9 \pm 1.5 \mathrm{~kg} / \mathrm{m}^{2}$; bench press strength: $1.3 \pm$ $0.2 \mathrm{~kg} / \mathrm{kg} \mathrm{BM}$; leg press strength: $3.4 \pm 0.9 \mathrm{~kg} / \mathrm{kg} \mathrm{BM}$ ) completed a supervised resistance training program in conjunction with a hypercaloric diet. The protocol lasted 6 weeks, and participants completed RMR assessments via IC pre-and post-intervention to obtain reference values. Existing RMR prediction equations based on body mass or fat-free mass were also evaluated. Equivalence testing was used to evaluate whether each prediction equation demonstrated equivalence with IC based on a $\pm 50 \mathrm{kcal} / \mathrm{d}$ equivalence region, and the confidence limits for the two-one-sided t-tests were calculated. Null hypothesis significance testing was performed, and Bland-Altman analyses were utilized alongside linear regression to assess the degree of proportional bias. RESULTS: IC RMR values increased by $165 \pm 97$ $\mathrm{kcal} / \mathrm{d}$. All prediction equations underestimated RMR changes, relative to IC, with magnitudes ranging from 75 to $132 \mathrm{kcal} / \mathrm{d}$, while also displaying unacceptable levels of negative proportional bias. Additionally, all prediction equations significantly differed from measured IC values, and no equation demonstrated equivalence with IC. CONCLUSION: These findings suggest the examined prediction equations are not acceptable for tracking RMR changes in resistance-trained males, within the context of the present study. The consistent underestimation of RMR changes indicates that the input variables, and their weights within the prediction equations, were insufficient to adequately explain the observed changes in RMR. 